Published in final edited form as:

Am J Ind Med. 2019 January ; 62(1): 59-68. doi:10.1002/ajim.22928.

\title{
Efficiency of autocoding programs for converting job descriptors into Standard Occupational Classification (SOC) codes
}

\author{
Skye Buckner-Petty, MPH ${ }^{1}$, Ann Marie Dale, PhD, OTR/L ${ }^{1}$, and Bradley A. Evanoff, MD, \\ $\mathrm{MPH}^{1}$ \\ ${ }^{1}$ Division of General Medical Sciences, Washington University School of Medicine, St. Louis, MO, \\ USA
}

\section{Abstract}

Background-Existing datasets often lack job exposure data. Standard Occupational Classification (SOC) codes can link work exposure data to health outcomes via a Job Exposure Matrix, but manually assigning SOC codes is laborious. We explored the utility of two SOC autocoding programs.

Methods-We entered industry and occupation descriptions from two existing cohorts into two publicly available SOC autocoding programs. SOC codes were also assigned manually by experienced coders. These SOC codes were then linked to exposures from the Occupational Information Network (O*NET).

Results-Agreement between the SOC codes produced by autocoding programs and those produced manually was modest at the 6-digit level, and strong at the 2-digit level. Importantly, O*NET exposure values based on SOC code assignment showed strong agreement between manual and autocoded methods.

Conclusion-Both available autocoding programs can be useful tools for assigning SOC codes, allowing linkage of occupational exposures to data containing free-text occupation descriptors.

\section{Keywords}

industry and occupation coding; job exposure matrix; NIOCCS; O*NET; SOCcer

Corresponding Author: Skye Buckner-Petty, MPH, Division of General Medical Sciences, Washington University School of Medicine, 4523 Clayton Avenue, Campus Box 8005, St. Louis, MO 63110, skye.skye@ wustl.edu.

Authors' contributions:

Skye Buckner-Petty designed and executed the analytic strategy for this study. He was also the primary writer of the manuscript. Ann Marie Dale conducted the manual job coding and assisted with writing. Bradley A. Evanoff provided guidance, assisted with writing, is the PI on the grant that funded this project, and is the PI on the grant that funded one of the two cohort studies that provided source data. All authors approved the final draft of the manuscript.

Institution and Ethics approval and informed consent: The analysis was completed using secondary data, which was de-identified; IRB review was not required.

Disclaimer: None

Disclosure: The authors declare no conflicts of interest. 


\section{INTRODUCTION}

Analyzing occupational risk factors is of great interest in epidemiological research. Working Americans spend a large portion of their day at the workplace ${ }^{1}$, therefore it is important to focus on determinants of health that are related to work and workplace behaviors. However, classifying jobs and identifying occupational risk factors can be challenging, especially when using pre-existing data. When this occupational information is available, it is most commonly in the form of free-text responses to open-ended questions regarding industry, job titles, and/or job tasks. In order to analyze these data, it is necessary to assign Standard Occupational Classification (SOC) codes to the free-text entries. The SOC system is used by federal statistical agencies in the United States for the purpose of collecting, calculating and disseminating data. These SOC codes have a hierarchical structure. The first two digits indicate a classification into one of 23 "major groups." There are 97 "minor groups" represented by the first four digits, and 461 "broad occupations" represented by the first five digits. Finally, six digits represent a "detailed occupation" classification, of which there are $840 .^{2}$ For research purposes, any of these classification levels can be used for studying associations between occupation and health outcomes and behaviors. The greater the number of digits indicated in the SOC codes, the higher the level of homogeneity of the characteristics in jobs represented by the code.

SOC codes can also be used to assign various job-level exposure estimates by linking job titles to job exposure matrices (JEMs). JEMs provide a source to obtain job related exposure data for existing studies that lack such data. JEMs are commonly used for studying chemical, electromagnetic, and noise exposures. However, JEMs can be useful to the study of a wide range of health outcomes. There has been recent interest in applying JEMs to study the effects of workplace physical demands on musculoskeletal disorders, pregnancy outcomes, hernias, and cardiovascular disease. ${ }^{3-7}$ Such job-related data can be obtained through The Occupational Information Network $\left(\mathrm{O}^{*} \mathrm{NET}\right)^{8}$ provided by the U.S. Department of Labor. O*NET is a publicly available database that contains a variety of descriptors of physical and mental demands related to SOC codes. Several recent studies have used O*NET-based physical exposure estimates to evaluate relationships between workplace exposures and chronic disease outcomes. ${ }^{9-11}$ One recent study showed the reliability of some $\mathrm{O} *$ NET-based force and repetition variables of the hand and wrist compared to similar directly observed measures, and demonstrated their ability to predict incident carpal tunnel syndrome. ${ }^{3}$

Manually assigning SOC codes is a time-consuming process; in large studies, this method can be infeasible. In addition, manual coding is not conducive to research reproducibility. Two publicly available autocoding programs have been created to alleviate this burden from researchers and facilitate large-scale studies that require occupation data (the US National Institute for Occupational Safety and Health has developed the NIOSH Industry and Occupation Computerized Coding System (NIOCCS) Version 3, and The National Institutes of Health provided Standardized Occupation Coding for Computer-assisted Epidemiological Research (SOCcer) Version 1). NIOCCS has been previously reviewed by Schmitz et al., ${ }^{12}$ and Weiss et al. ${ }^{13}$ to show feasibility of use, however additional testing in various datasets is necessary to demonstrate performance capabilities. This program has recently been used to 
categorize occupational information in Behavioral Risk Factor Surveillance System (BRFSS) data. ${ }^{14,15}$ Details of SOCcer are described by Russ ${ }^{16}$ along with preliminary testing. However, to our knowledge, SOCcer has not been formally tested by researchers unaffiliated with the development team. Furthermore, no studies have compared the two programs using data from published cohorts. The purpose of this study was to test and compare the performance of both job title autocoding programs using data from two existing epidemiological studies. We compared agreement between SOC codes from the coding programs to that of expert manual coders. We also compared agreement between autocoding and manual coding on the subsequent exposure values obtained when using assigned SOC codes to extract six relevant occupational physical exposures from O*NET.

\section{METHODS}

\section{Study population}

Two cohorts from previously completed studies were used for the current study. The secondary data from these cohorts was de-identified; IRB review was not required. Cohort 1 consisted of pooled data from six prospective studies of workplace risk factors for upper extremity musculoskeletal disorders. Details of the pooled cohort have been described elsewhere. ${ }^{17,18}$ Briefly, study participants were fulltime male and female employees, 18 years of age or older, who worked in a variety of industries including manufacturing, production, service, and construction. In total, 4,321 workers were recruited across the six study sites and followed between 2001 and 2010.

Cohort 2 consists of data from a cross-sectional, telephone-based survey that was conducted as part of the Supports at Home and Work for Maintaining Energy Balance (SHOW-ME) study. The SHOW-ME study was designed to examine the associations between residential and worksite environmental and policy influences and energy balance behaviors and outcomes. There were 2,015 participants in this cohort who were 21-65 years of age, and employed at least 20 hours per week. Further details of the SHOW-ME study are described elsewhere. ${ }^{19}$

\section{Manual job coding}

From cohort 1, information about each worker's current job was collected at baseline and follow-up including job title, company name, and work-related tasks. The last known job for each subject was used for this study as this provided a greater number of job titles than baseline data. In cohort 2, participants were asked to provide the name and street address of their primary workplace. They then were asked what kind of work they do (occupation) and in what type of business or industry. Using the available information from both cohorts, SOC codes were assigned to each worker using the job title selection feature provided by the O*NET online database ${ }^{8}$ and selecting the occupational code that best matched the primary tasks and employer information. Standard Industrial Classification (SIC) codes and National American Industry Classification System (NAICS) codes were assigned in a similar fashion after using ReferenceUSA ${ }^{20}$ to look up the employers of each worker. SOC, SIC and NAICS codes were independently assigned by two raters, with differences resolved by consensus. $\mathrm{O} * \mathrm{NET}$ assigns job exposure information for SOC codes at the detailed occupation level. 
Since linking free text job titles to O*NET physical exposures was the primary motivation for assigning SOC codes in both cohorts, the availability of exposure values influenced the coding decisions. Only subjects with sufficient job information that allowed for successful manual SOC, SIC and NAICS code assignments were included in the current study.

\section{NIOCCS autocoding}

NIOCCS is capable of assigning SOC codes using a combination of industry (input as freetext or NAICS codes) and occupation free-text. The SOC codes presented in the output can range in level of detail from 2 to 6 digits. Each code is provided with a score to indicate the level of confidence of the assignment. These scores will range from $90 \%$ to $100 \%$; codes with scores less than $90 \%$ are not included in the output. Self-reported occupation free-texts and manually assigned NAICS codes from both cohorts were input into the NIOCCS system. This was then repeated except omitting the NAICS codes.

\section{SOCcer autocoding}

SOCcer codes job descriptions to the SOC 2010 classification system as described in Russ. It allows for batch input of occupation, SIC code, and job task; partial information is allowed. For each row of job-descriptors, the system outputs 10 six-digit SOC codes, each of which is assigned a confidence score representing the estimated probabilities (computed from logistic regression) that an expert reviewer would have selected that SOC code. For convenience, the paper will refer to scores from SOCcer and NIOCCS with the same terminology, although their values are not directly comparable. Unlike NIOCCS, SOCcer will present SOC code options for every entry regardless of confidence levels. It is the user's responsibility to omit codes with low scores. Self-reported occupation based on free text responses and manually assigned SIC codes from both cohorts were used to produce SOCcer output. This was then repeated except with the SIC codes omitted. The SOCcer produced SOC code with the highest confidence score for each subject was used for analyses. In the presence of ties, the first code presented was used.

\section{O*NET exposures}

Using the SOC codes assigned to each subject by all three coding methods (manual, SOCcer, and NIOCCS), physical work exposure variables were extracted from $\mathrm{O} * \mathrm{NET}$ databases. A total of 6 items describing physical workplace demands were selected from three different $\mathrm{O} *$ NET databases (work activities, work context, and work abilities). The selected physical exposure items were those used in past studies of the two cohorts, and included (a) dynamic strength, (b) static strength, (c) handling and moving objects, (d) time spent making repetitive movements, (e) importance of using computers, and (f) performing general physical activities. The values for each of these exposures were assigned to the participant's SOC codes. O*NET exposures are classified by 8-digit SOC codes. Since both autocoding programs produce up to 6-digit SOC codes, ".00" was attached to each code prior to extracting the $\mathrm{O}^{*} \mathrm{NET}$ values. SOC codes from NIOCCS that were presented with fewer than 6-digits were not linked to O*NET data. 


\section{Analyses}

Cohen's Kappa statistic was used to measure agreement between SOC codes produced manually and by the autocoding programs. Agreement was computed for 6-digit (Detailed Occupation), and 2-digit (Major Group) SOC codes. Agreement between NIOCCS and manual codes was assessed with and without inputted NAICS codes. Agreement between SOCcer and manual codes was assessed with and without inputted SIC codes. Furthermore, SOC agreement was assessed at various levels of confidence scores for both programs. We assessed the distributions of the confidence scores to determine appropriate stratifications. We also assessed the overall effectiveness of both programs by computing the percentage of each cohort that was accurately autocoded. Cohen's Kappa was also used to measure agreement directly between NIOCCS and SOCcer with and without inputted industry codes.

Intraclass correlations (ICC) were computed to measure agreement between the $\mathrm{O} * \mathrm{NET}$ values linked to SOC codes produced manually versus those produced by autocoding programs with industry codes and occupation text included as inputs. The frequency of SOC codes produced by autocoding programs that had available $\mathrm{O}$ *NET data was also captured. For interpretation of the kappa and ICC values, we followed the guidelines set by Cicchetti ${ }^{21}$ and considered values less than 0.40 as poor; between 0.40 and 0.59 as fair; between 0.60 and 0.74 as good; and between 0.75 and 1.00 as excellent. All analyses were conducted using $\mathrm{R}$ version 3.3.2.2

\section{RESULTS}

The inclusion criteria were met by 1,823 subjects ( 3 out of 6 study sites) in cohort 1 and 1,496 subjects in cohort 2 . In cohort 1 , three of the study sites reported job tasks but not job titles. Additional subjects were dropped from both cohorts due to a missing manual SOC, SIC, or NAICS code assignment. Incomplete manual codes resulted from missing or ambiguous job information. Table I shows the frequencies of the 23 SOC major groups (defined by the manual SOC codes). Both cohorts have a wide variety of job types. Cohort 2 includes subjects in all SOC major groups except "Military Specific Occupations." Cohort 1 includes subjects in all SOC major groups except "Military Specific Occupations" and "Farming, Fishing, and Forestry Occupations." Cohort 2 had more job diversity than Cohort 1, but had relatively large proportions in "Office and Administrative Support Occupations" (17.8\%), "Education, Training, and Library Occupations" (14.2\%) and "Healthcare Practitioners and Technical Occupations" (12.5\%). Cohort 1 had a large proportion in "Production Occupations" (33.1\%). Cohort 1 and cohort 2 contained 263 and 322 unique 6digit SOC codes respectively.

Table II shows agreement levels for SOC codes assigned by autocoding programs with manually assigned SOC codes. SOCcer codes were stratified into three categories based on confidence scores: less than $0.2,0.2$ to 0.4 , and greater than 0.4 . For both cohorts, roughly a third of the codes fit into each of these strata when SIC codes were included as an input. When SIC codes were omitted, the majority of codes had a confidence score less than 0.2 (cohort $1=58.7 \%$; cohort $2=62.0 \%$ ). Confidence scores in NIOCCS ranged from 90 to 100 ; however more than half of the available scores were 100 for both cohorts, with and without NAICS inputted; so we created two strata: scores equal to 100 versus scores less than 100. 
NIOCCS did not produce SOC codes for all entries. Most entries were coded when NAICS codes were inputted (cohort $1=84.6 \%$; cohort $2=79.5 \%$ ). However, few were coded when NAICS codes were omitted (cohort $1=31.4 \%$; cohort $2=45.1 \%$ ).

Both autocoding programs consistently showed increased agreement with manually assigned SOC codes for higher confidence scores regardless of cohort and inputs. Overall, NIOCCS had excellent agreement with 2-digit SOC codes (kappa $=0.75-0.82)$ and fair to good agreement for 6-digit SOC codes (kappa $=0.41-0.63$ ). SOCcer had fair to good agreement with 2-digit SOC codes (kappa $=0.55-0.70)$ and poor to fair agreement with 6-digit SOC codes (kappa $=0.30-0.41$ ). Within the subset of jobs that were coded by both programs, NIOCCS showed stronger agreement than SOCcer for both cohorts.

NIOCCS and SOCcer had similar levels of effectiveness when industry codes were included as inputs. However, when industry was omitted, NIOCCS's effectiveness drastically decreased. With industry included, NIOCCS's effectiveness ranged from $64.0 \%$ to $67.4 \%$ for 2-digit codes, and $33.6 \%$ to $44.4 \%$ for 6-digit codes. However, with industry omitted, NIOCCS's effectiveness ranged from $24.3 \%$ to $37.8 \%$ for 2-digit codes, and $16.1 \%$ to $28.7 \%$ for 6-digit codes. On the contrary, SOCcer's effectiveness only decreased slightly when industry was omitted. With industry included, SOCcer's effectiveness ranged from $62.4 \%$ to $72.3 \%$ for 2-digit codes, and $31.3 \%$ to $41.8 \%$ for 6 -digit codes. With industry omitted, SOCcer's effectiveness ranged from $61.2 \%$ to $68.2 \%$ for 2-digit codes, and $30.6 \%$ to $40.5 \%$ for 6-digit codes.

As previously mentioned, the confidence scores from both programs are not directly comparable. However, Figure1 shows agreement levels at various confidence score cutoffs such that SOCcer scores and NIOCCS scores are aligned by the proportion of the cohort that is coded. NIOCCS had higher agreement than SOCcer in both cohorts for all comparable confidence levels.

Table III shows agreement between the SOC codes produced by both autocoding programs. When industry codes were included as inputs, we observed poor to fair agreement for 6-digit SOC codes (kappa $=0.37-0.49$ ) and good to excellent agreement for 2-digit SOC codes (kappa $=0.60-0.75)$. When industry was omitted, we observed fair to good agreement with 6-digit SOC codes and excellent agreement for 2-digit SOC codes (kappa $=0.77-0.80$ ).

Table IV shows the agreement of O*NET physical exposure variables for SOC codes from autocoding programs compared to those of manually coded SOC codes. Agreement was good to excellent for most comparisons in both programs. Again, higher confidence levels led to stronger agreement. Consequently, both programs produced some codes that were not linkable to O*NET exposures. NIOCCS was slightly more likely to produce SOC codes that did not have exposure data available in $\mathrm{O} *$ NET. For cohort $1,65.1 \%$ of the NIOCCS produces codes versus $75.2 \%$ of the SOCcer produced codes were linkable to O*NET exposures. Likewise, for cohort $2,82.4 \%$ of the NIOCCS produced codes versus $86.8 \%$ of the $\mathrm{SOCcer}$ produced codes were linkable to $\mathrm{O}$ *NET exposures. 


\section{DISCUSSION}

We evaluated the performance abilities of SOCcer and NIOCCS to produce SOC codes from self-reported text entries in comparison to SOC codes manually coded by researchers in two published cohort studies. We also evaluated the agreement of O*NET exposure values linked to the aforementioned SOC codes. Both programs demonstrated to be useful tools. NIOCCS had slightly better agreement with manually coded SOC codes than SOCcer when industry codes were included. However, in the absence of industry codes, SOCcer was much more effective. Overall, both programs performed modestly for 6-digit SOC codes, with agreement to manually produced codes ranging from poor to good. However, much stronger agreement was observed for 2-digit SOC codes; similarly, strong agreement was observed between $\mathrm{O}$ *NET exposures linked to the 6-digit SOC codes produced manually versus those produced by the autocoding programs. This shows that even when disagreement was found for 6-digit SOC codes, the discrepant coding assignments were quite similar in terms of the job classifications and the job-exposures. Therefore, for most research purposes, these discrepancies are likely to be inconsequential.

The agreement that we observed between SOCcer codes and manually coded SOC codes closely mirrored the results from Russ et al at the 2-digit and the 6-digit levels. ${ }^{16}$ A previous evaluation of an earlier version of NIOCCS, by Schmitz and Forst, made comparisons at the 2-digit and 4-digit levels ${ }^{12}$. Their findings at the 2-digit level were close to our results. The strength of agreement that they found at the 4-digit level was weaker than that of the 2-digit level, but still stronger than what we found at the 6-digit level. This suggests that there is reasonable agreement across various datasets between these two autocoding programs. To our knowledge, no previous papers have evaluated agreement between job-exposures estimates made based on autocoded and manually coded job titles. This latter comparison is important, since many researchers seek to code job titles in order to make estimates of workrelated exposures. Our finding of good to excellent agreement between exposure values assigned based on manual coding vs. autocoding may encourage more researchers to assign workplace exposures based on job title information in a variety of epidemiology studies that would otherwise lack work exposure data.

Both autocoding programs have additional features that were not evaluated in this study. Deciding which program to use will depend on aligning the data with the available features. NIOCCS accepts industry in the form of free text as well as NAICS codes; while SOCcer requires industry to be included as SIC codes. Often, data will include industries that are not already coded, thus making NIOCCS the appropriate choice. Conversely, SOCcer has the ability to process free-text job tasks, so if data contains both job titles and tasks, or have subjects that provide tasks without titles, then SOCcer may be the more appropriate choice. Also, since SOCcer was more effective than NIOCCS when industry was omitted, SOCcer should be used in situations where industry is not available.

Both program performed slightly better in cohort 2 than in cohort 1 . A qualitative review showed that cohort 1 contained more ambiguous free-text entries than cohort 2 . Taking measures to avoid ambiguous occupation text entries should be considered during data collection. 
SOCcer lists 10 SOC codes for every data entry providing additional flexibility for the user. However, users are cautioned to review the confidence scores. Even though SOC codes are provided for every entry, a low score may indicate that the associated SOC code is not reliable and should not be used. A priori decisions should be made regarding procedures for using SOCcer output including determining a lowest acceptable confidence score. Also, it must be decided whether to use only the SOC code with highest score, or to consider all the SOC codes with scores that meet the specified cutoff. In practice, it may be beneficial to manually code a random subset of data to get a sense of how well SOCcer performs at various confidence scores. Further investigation in this area may help inform decision making for SOCcer users.

Both autocoding programs may leave a subset of data without adequate SOC codes. In these instances additional manual coding may be necessary. Both programs provide an interface that assists users to manually code any non-coded entries, and to review codes with low confidence scores (or flagged for suggested review in NIOCCS). However, in some circumstances any amount of manual coding may be infeasible or undesirable. Researchers may choose to exclude data that are not supplied adequate codes from a coding program, however, caution should be taken as this may lead to data that are missing not at random. That is, there may be some meaningful differences between subjects that are successfully assigned SOC codes versus those that are not. It should also be noted that availability of O*NET exposure data influenced the manual coding process in both cohorts of the present study. This likely caused some attenuation in agreement since availability of O*NET exposures is not considered by the autocoding programs. Also, since data collection methods, job diversity, and types of job descriptors all differ among datasets, the results of the current study may not be typical.

The use of JEMs in epidemiology is growing. Primary data collection can be very expensive, thus, the ability to combine information from JEMs with pre-existing data is often attractive. Furthermore, JEMs allow the ability to obtain retrospective exposure data, whereas it is impossible to directly observe such exposures, and self-reported exposures from past jobs may suffer from recall bias or random misclassification. The ability to convert free-text job titles into SOC codes with autocoding programs provides an efficient process for using JEMs.

SOCcer and NIOCCS are powerful tools for assigning SOC codes to free-text occupation entries. This helps facilitate a key step in epidemiological studies of occupational related risk factors. Manual coding can be very time consuming and often times infeasible for large studies. Furthermore, manual coding is an inherently less consistent method than using autocoding programs. Therefore, as the use of SOC codes in research continues to grow, autocoding will likely become standard practice. With this eminent shift, further testing of these autocoding programs is urged.

\section{Acknowledgements:}

The authors would like to thank the following groups and individuals whose contributions were necessary for this research: NIOSH Division of Surveillance, Hazard Evaluations, and Field Studies for providing guidance and troubleshooting for use of NIOCCS, Dr. Daniel Russ from the NIH for providing guidance and troubleshooting for 
use of SOCcer, and investigators from the SHOW-ME study (U54 CA155496) and the NIOSH Upper Extremity Musculoskeletal Disorders Consortium (R01OH009712) for access to data from these two studies.

Funding: Grant sponsor: American National Institute for Occupational Safety and Health; Grant number: R01OH011076

\section{References}

1. Bureau of Labor Statistics. Average hours per day spent in selected activities on days worked by employment status and sex, 2017 annual averages. In. Washington, DC: U.S. Bureau of Labor Statistics; 2017.

2. Bureau of Labor Statistics. Standard Occupational Classification. 2018; https://www.bls.gov/soc/.

3. Dale AM, Ekenga CC, Buckner-Petty S, et al. Incident CTS in a large pooled cohort study: associations obtained by a Job Exposure Matrix versus associations obtained from observed exposures. Occup Environ Med. 2018;75(7):501-506. [PubMed: 29599164]

4. Mediouni Z, Bodin J, Dale AM, et al. Carpal tunnel syndrome and computer exposure at work in two large complementary cohorts. BMJ open. 2015;5(9):e008156.

5. Solovieva S, Pehkonen I, Kausto J, et al. Development and validation of a job exposure matrix for physical risk factors in low back pain. PLoS One. 2012;7(11):e48680. [PubMed: 23152793]

6. Rubak TS, Svendsen SW, Soballe K, Frost P. Total hip replacement due to primary osteoarthritis in relation to cumulative occupational exposures and lifestyle factors: A nationwide nested casecontrol study. Arthritis Care Res. 2014;66(10):1496-1505.

7. Svendsen SW, Johnsen B, Fuglsang-Frederiksen A, Frost P. Ulnar neuropathy and ulnar neuropathylike symptoms in relation to biomechanical exposures assessed by a job exposure matrix: a triple case-referent study. Occup Environ Med. 2012;69(11):773-780. [PubMed: 22843442]

8. Occupational Information Network, U.S. Department of Labor/Employment and Training Administration. O*NET OnLine website. 2018; http://www.onetonline.org/.

9. Evanoff B, Zeringue A, Franzblau A, Dale AM. Using job-title-based physical exposures from O*NET in an epidemiological study of carpal tunnel syndrome. Hum Factors. 2014;56(1):166-177. [PubMed: 24669551]

10. Dale A, Zerinque A, Harris-Adamson C, et al. General Population Job Exposure Matrix Applied to a Pooled Study of Prevalent Carpal Tunnel Syndrome. Am J Epidemiol. 2015;181(6):431-439. [PubMed: 25700886]

11. Dembe AE, Yao X, Wickizer TM, Shoben AB, Dong XS. Using O*NET to Estimate the Association Between Work Exposures and Chronic Diseases. Am J Ind Med. 2014;57(9):10221031. [PubMed: 24842122]

12. Schmitz M, Forst L. Industry and occupation in the electronic health record: an investigation of the National Institute for Occupational Safety and Health Industry and Occupation Computerized Coding System. JMIR medical informatics. 2016;4(1).

13. Weiss N, Cooper S, Socias C, Weiss R, Chen V. Coding of Central Cancer Registry Industry and Occupation Information: The Texas and Louisiana Experiences. Journal of registry management. 2015;42(3):103-110. [PubMed: 27028094]

14. Dodd KE, Mazurek JM. Asthma among employed adults, by industry and occupation-21 states, 2013. 2016.

15. O'Halloran AC, Lu PJ, Williams WW, et al. Influenza vaccination among workers-21 U.S. states, 2013. Am J Infect Control. 2017;45(4):410-416. [PubMed: 28364911]

16. Russ DE, Ho K-Y, Colt JS, et al. Computer-based coding of free-text job descriptions to efficiently identify occupations in epidemiological studies. Occup Environ Med. 2016:oemed-2015-103152.

17. Rempel D, Gerr F, Harris-Adamson C, et al. Personal and Workplace Factors and Median Nerve Function in a Pooled Study of 2396 US Workers. J Occup Environ Med. 2015;57(1):98-104. [PubMed: 25563546]

18. Dale AM, Harris-Adamson C, Rempel D, et al. Prevalence and incidence of carpal tunnel syndrome in US working populations: pooled analysis of six prospective studies. Scand J Work Environ Health. 2013;39(5):495-505. [PubMed: 23423472] 
19. Dale AM, Enke C, Buckner-Petty S, et al. Availability and Use of Workplace Supports for Health Promotion Among Employees of Small and Large Businesses. Am J Health Promot. 2018:890117118772510.

20. ReferenceUSA. 2017 http://www.referenceusa.com/Home/Home Accessed March 082017.

21. Cicchetti DV. Guidelines, criteria, and rules of thumb for evaluating normed and standardized assessment instruments in psychology. Psychol Assess. 1994;6(4):284.

22. R: A Language and Environment for Statistical Computing [computer program]. Version 3.3.2. Vienna, Austria: R Foundation for Statistical Computing; 2016. 


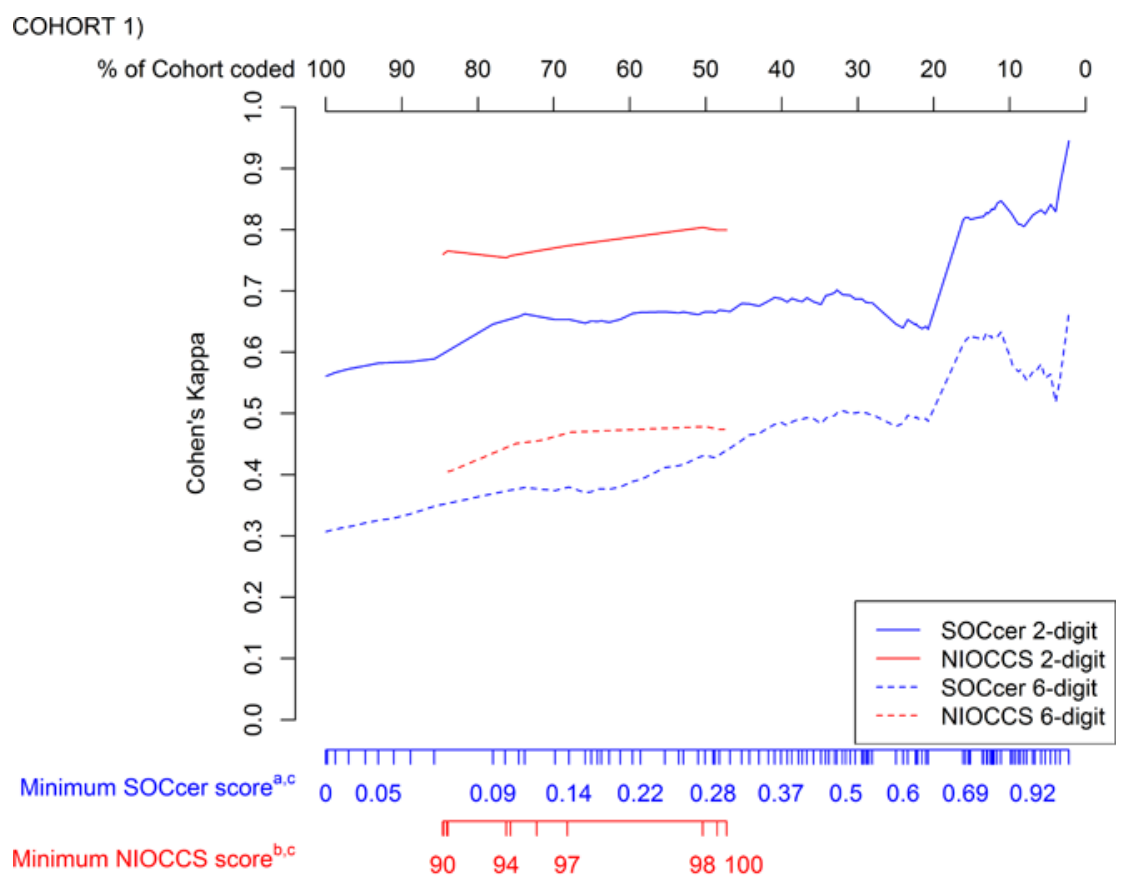

COHORT 2)
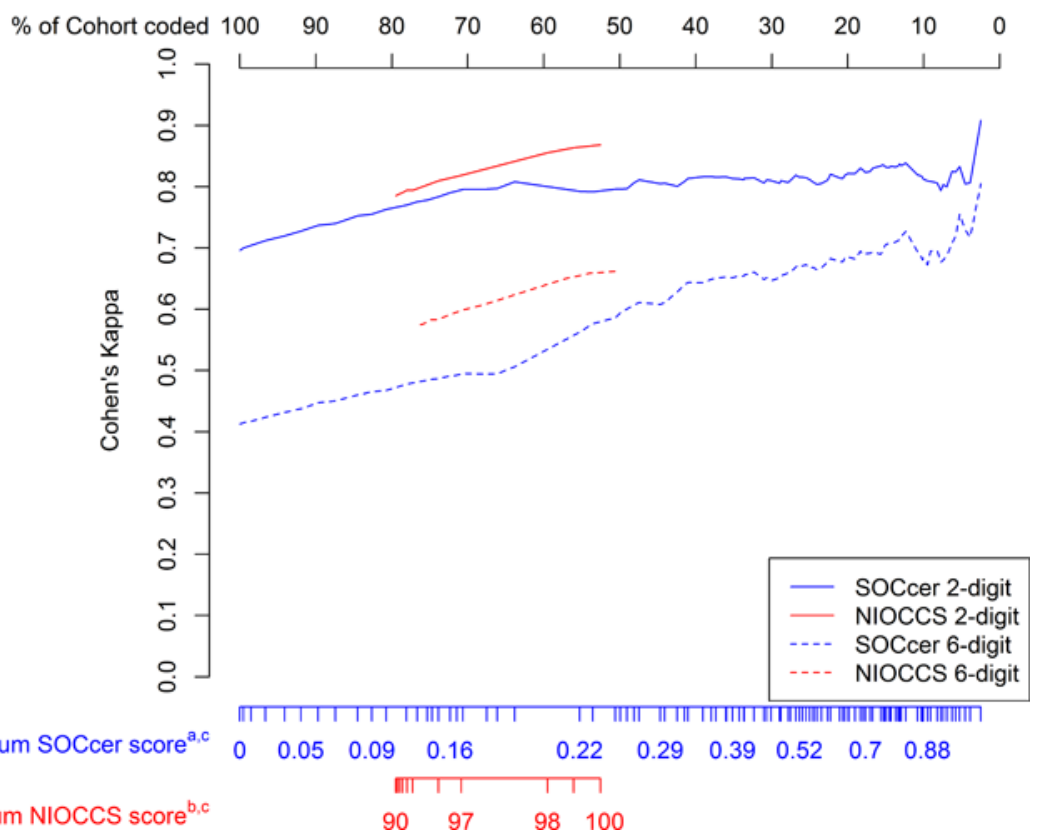

Figure 1:

Agreement Between Autocoded and Manually Coded SOC Codes Based on Minimum Allowed Confidence Scores From Pooled Upper Extremity Study (Cohort 1), United States, 2001 - 2010, and From the Show-ME Study (Cohort 2), Missouri, 2012 - 2013

NIOCCS (NIOSH Industry and Occupation Computerized Coding System); SOC (Standard Occupational Classification); SOCcer (Standardized Occupation Coding for Computerassisted Epidemiological Research)

a SOCcer scores range from 0-1 
${ }^{\mathrm{b}}$ NIOCCS scores range from $90-100$ (SOC codes with scores less than 90 are omitted by the program)

c SOCcer and NIOCCS scores are aligned based on the proportion of the cohort that is autocoded at each minimum threshold

Industry codes and occupation text included for both autocoding programs 


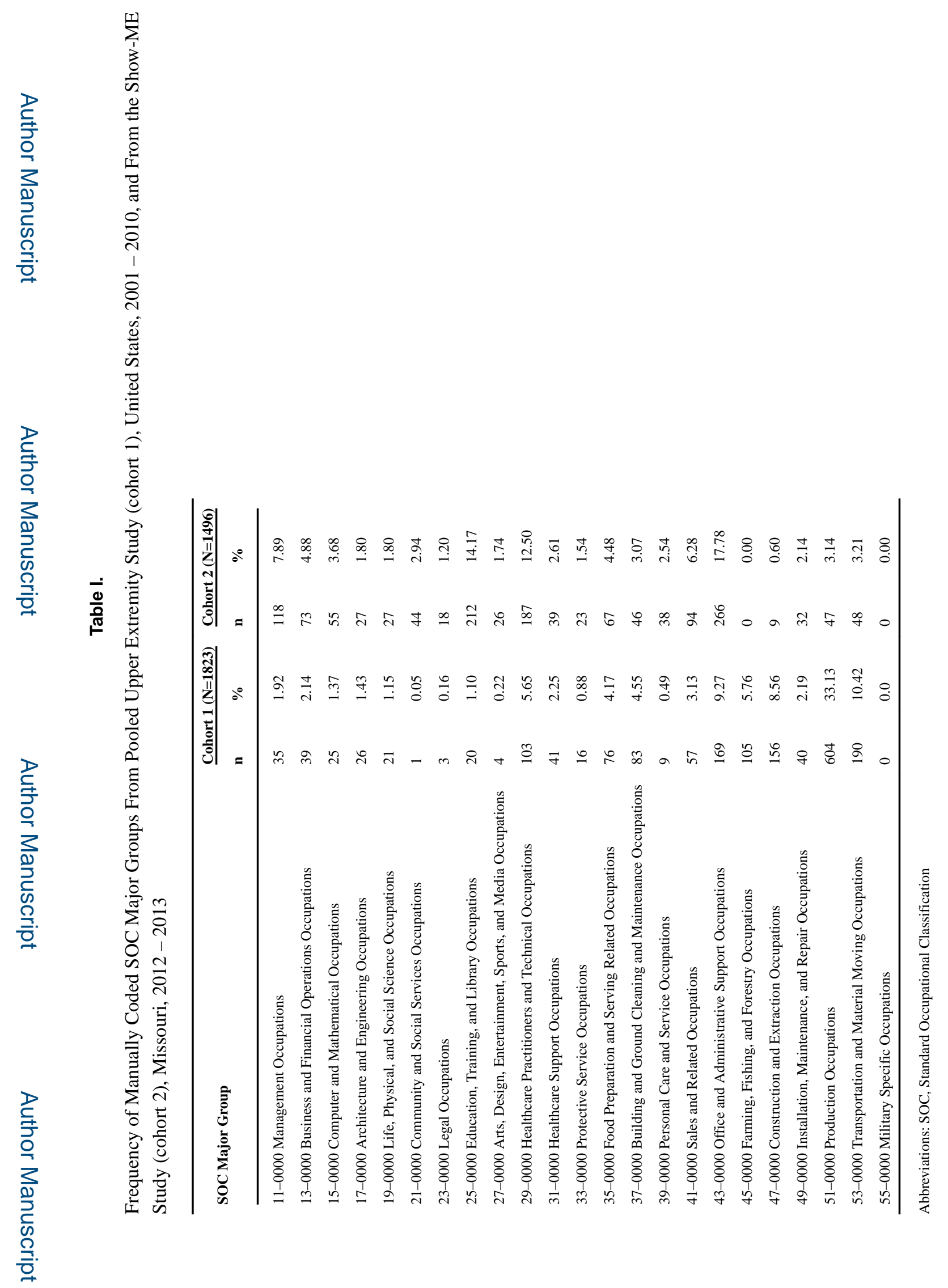

Am J Ind Med. Author manuscript; available in PMC 2020 January 01. 
Buckner-Petty et al.

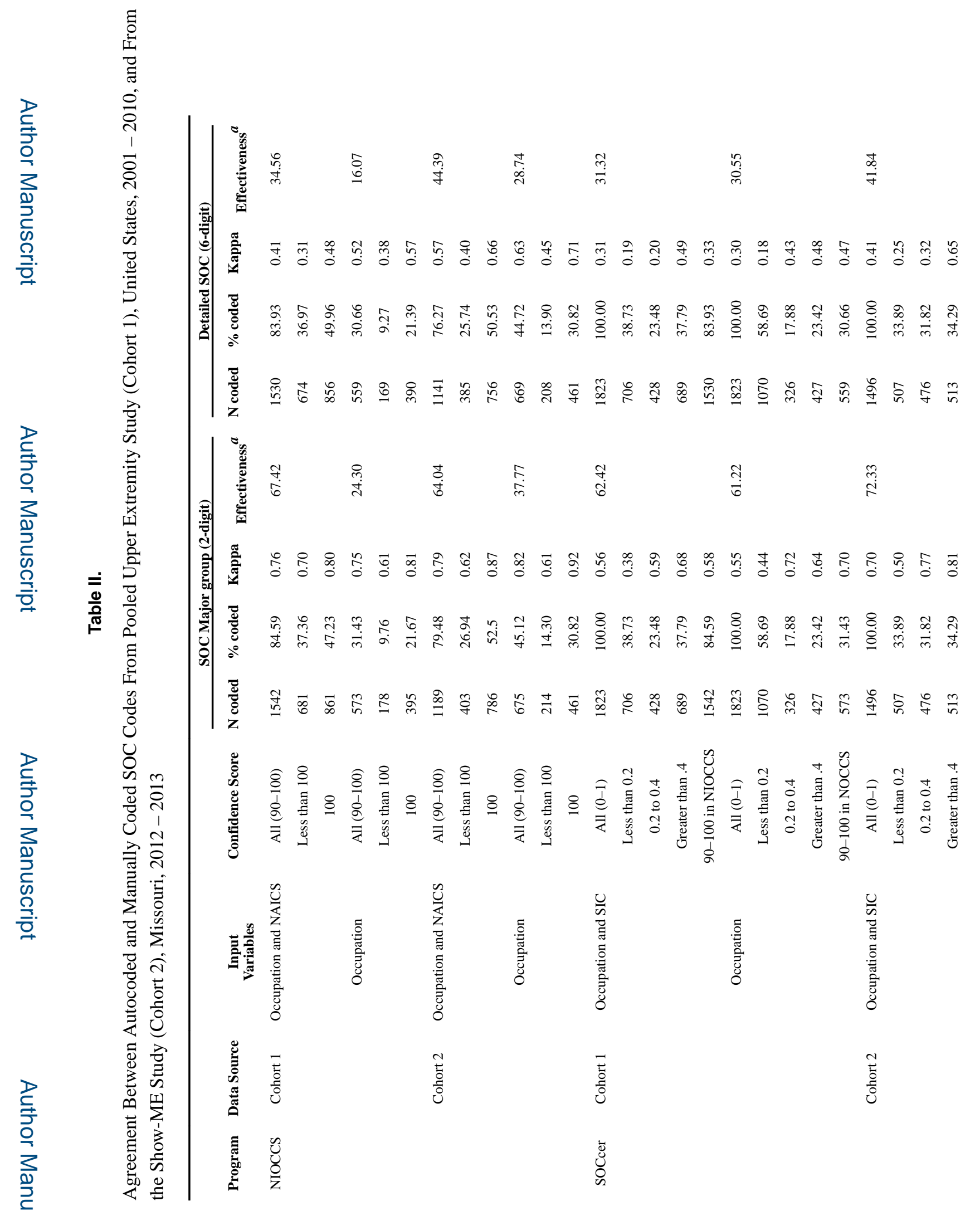

Am J Ind Med. Author manuscript; available in PMC 2020 January 01. 


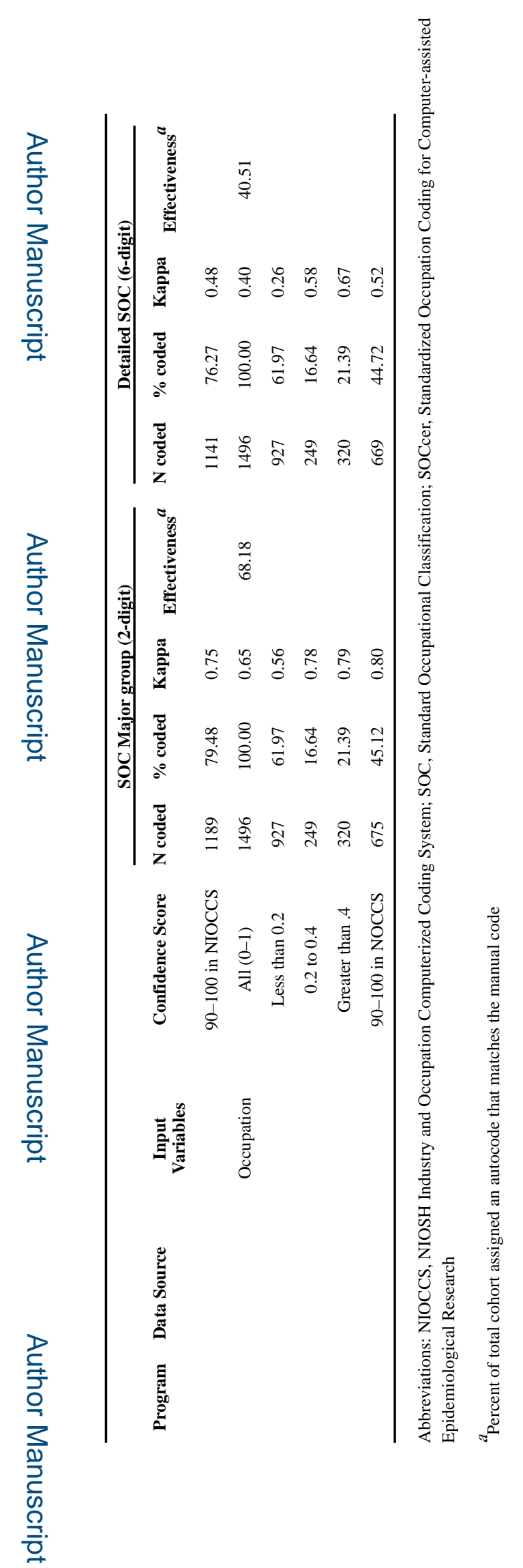




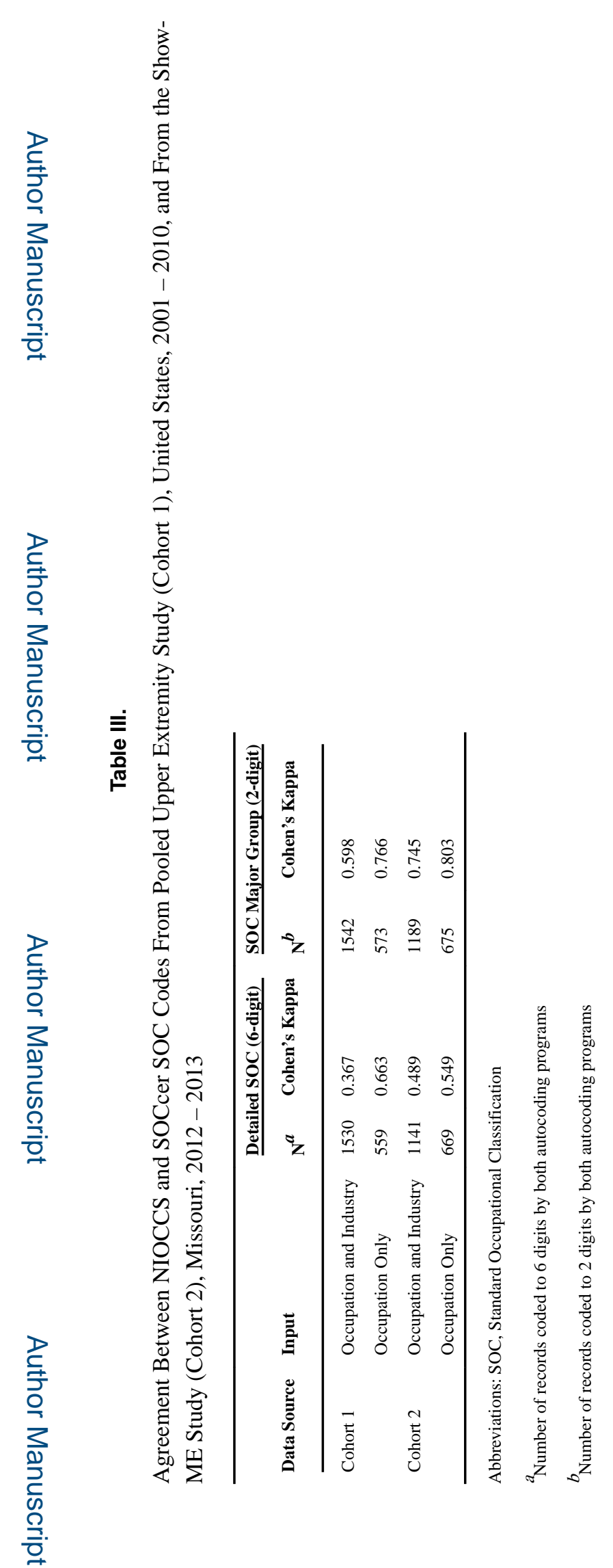




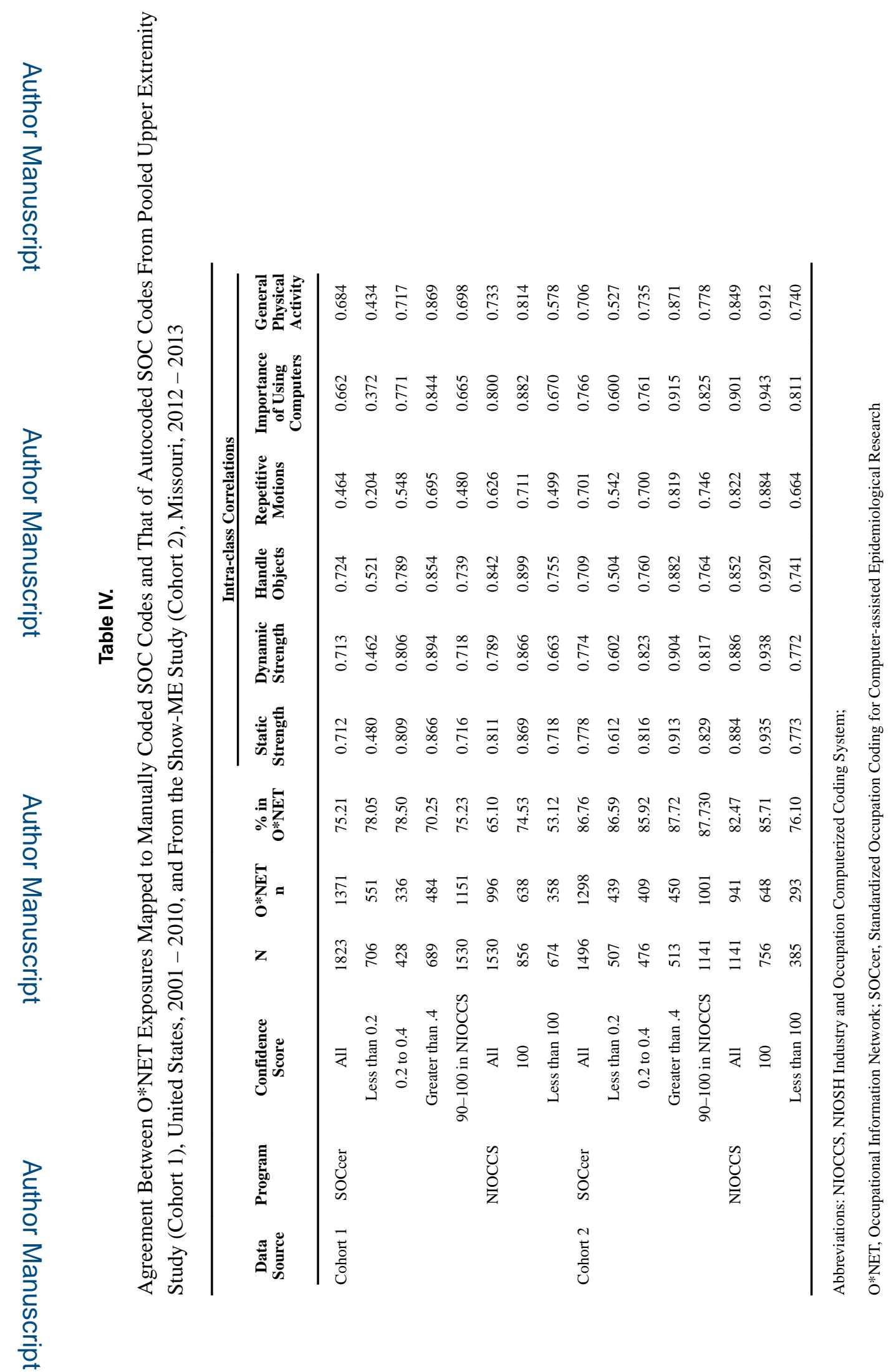

Am J Ind Med. Author manuscript; available in PMC 2020 January 01. 